\title{
In vivo effects of curcumin and deferoxamine in experimental endometriosis
}

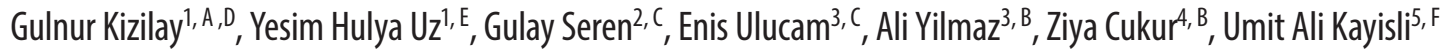 \\ ${ }^{1}$ Department of Histology and Embryology, Trakya University Faculty of Medicine, Edirne, Turkey \\ 2 Department of Analytical Chemistry, Trakya University Faculty of Pharmacy, Edirne, Turkey \\ ${ }^{3}$ Department of Anatomy, Trakya University Faculty of Medicine, Edirne, Turkey \\ ${ }^{4}$ Experimental Animal Center, Trakya University, Edirne, Turkey \\ ${ }^{5}$ Department of Obstetrics and Gynecology, Morsani, College of Medicine, South Florida, USA \\ A - research concept and design; $\mathrm{B}$ - collection and/or assembly of data; $\mathrm{C}$ - data analysis and interpretation; \\ $D$ - writing the article; $E$ - critical revision of the article; $F$ - final approval of article
}

\section{Address for correspondence}

Gulnur Kizilay

E-mail: gulnurkizilay@trakya.edu.tr

Funding sources

This study was supported by Trakya University Research Center, Edirne, Turkey.

\section{Conflict of interest}

none declared

Received on January 17, 2014

Revised on August 08, 2014

Accepted on September 18, 2014
DOI

10.17219/acem/31186

\section{Copyright}

Copyright by Author(s)

This is an article distributed under the terms of the

Creative Commons Attribution Non-Commercial License

(http://creativecommons.org/licenses/by-nc-nd/4.0/)

\section{Abstract}

Background. Endometriosis is one of the most common chronic gynecological diseases.

Objectives. The aim of the study was to examine the effects of curcumin and/or deferoxamine on cell proliferation in a rat model of endometriosis.

Material and methods. Thirty female 12-week-old albino Wistar rats, weighing 200-250 g, were used in this study. All the rats underwent ovariectomy and $0.1-\mathrm{mg} \beta$-estradiol 17-valerate pellets were placed intraperitoneally. An experimental model of endometriosis was created in all the animals. To create the experimental model, an approximately 1-cm long section of the uterus was taken, primarily from the right horn of the uterus. Autologous fragments were then placed between the peritoneum and muscle. The animals were divided into 3 groups: Group A, treated only with the vehicle used for curcumin and deferoxamine; group B, treated with curcumin (100 mg/kg body weight); and group C, treated with deferoxamine + curcumin (100 mg/kg body weight). After biopsy samples were obtained, the sections were stained with hematoxylin and eosin. Immunostaining for cytokeratin-7 and proliferating cell nuclear antigen (PCNA) was performed. Blood iron levels were measured using a Perkin Elmer AAnalyst 800 Atomic Absorption Spectrophotometer.

Results. The endometrial implant size increased in Group A, but treatment with curcumin $(p=0.01)$ and deferoxamine + curcumin $(p=0.007)$ reduced the implant size. In ectopic endometrial epithelial cells, there were significant decreases in PCNA immunoreactivity between groups $A$ and $B(p=0.044)$ and between groups $A$ and $C(p=0.033)$.

Conclusions. Treatment with curcumin alone and/or in combination with deferoxamine contributed to a reduction in implant size and cell proliferation in a rat endometriosis model. Iron-chelating agents may act in the same manner when used in women with endometriosis; however, further studies from different perspectives are still needed.

Key words: curcumin, endometriosis, deferoxamine, PCNA 
Endometriosis is one of the most common chronic gynecological diseases; it causes infertility, pelvic pain, dyspareunia and dysmenorrhea. The pathogenesis of this disorder remains unknown. ${ }^{1}$ Histologic and cellular changes observed in eutopic and ectopic endometrial tissues from experimentally induced endometriosis are almost identical to those observed in women with endometriosis. ${ }^{2-4}$ Iron levels are reportedly increased in women with endometriosis and in research animals with experimentally induced endometriosis. ${ }^{5-7}$ Iron plays an important role in the progression of endometriosis because it affects cell proliferation. ${ }^{6,7}$

Deferoxamine is an iron-chelating agent that is used to remove iron from the blood. Treatment with deferoxamine or with curcumin has produced significant results for some patients with endometriosis, pancreatic cancer and $\beta$-thalassemia. ${ }^{6,8-10}$

Curcumin is the active agent of the turmeric plant (Curcuma longa, turmeric, saffron root, turmeric). Turmeric is a perennial herbaceous plant with yellow flowers and large leaves that belongs to the ginger family and is widely grown in China and India. ${ }^{11,12}$ Curcumin has been used as a coloring agent in many foods, cosmetics and drugs, and is known to have antioxidant, anticarcinogenic, antiatherogenic and anti-inflammatory actions in addition to its iron-chelating effects. ${ }^{11,12}$ Its anti-inflammatory effects (as an inhibitor of NF-kB) have attracted increasing attention in recent years. Curcumin forms $\mathrm{Fe}^{+3}$-curcumin complexes by binding $\mathrm{Fe}^{+3}$, which increases its known effects. Its chemical binding capacity is greater for $\mathrm{Fe}^{+3}$ than $\mathrm{Fe}^{+2}$, depending on the dose and time of application. ${ }^{10}$

The present study investigates the impact of curcumin, alone and in combination with deferoxamine, on cell proliferation and/or implant growth in endometriotic tissues in in vivo experiments, with the aim of establishing the basis for a novel therapeutic strategy for the treatment of endometriosis.

\section{Material and methods}

\section{The animals and surgical procedures}

After the experimental procedures were approved by the local animal ethics committee, 30 female albino Wistar rats (12 weeks old, weighing 200-250 g) were obtained from the Experimental Animal Center of Trakya University. The initial body weights of the animals were measured. All the animals underwent ovariectomies (to eliminate estrogen from the ovaries) under ketamine- and xylazine-induced anesthesia (respectively: Alfamine, Alfasan International BV, Woerdam, The Netherlands; and Rompun, Bayer AG, Leverkusen, Germany). Next, 21-day release 0.1-mg $\beta$-estradiol 17-valerate pellets (E-203, Innovative Research of America, Sarasota, USA) were placed intraperitoneally. All the animals were examined at the same stage of the es- trous cycle by waiting 5 days after this procedure. Thereafter, an experimental model of endometriosis was created surgically using the method described by Rezende et al. ${ }^{4}$ Briefly, to create the experimental model, an approximately 1-cm-long section of the uterus was removed, primarily from the right horn of the uterus $1 \mathrm{~cm}$ above the cervix, and the cylinder-shaped section of the uterus was flattened. The myometrium was split from the endometrium under a microscope, and the dimensions of the 4 endometrial implants thus obtained were measured. Four implants were placed between the peritoneum and muscle. Ten days after this process, 2 test animals were checked, and it was confirmed that the endometriosis model had been created macroscopically and microscopically.

After the establishment of surgically induced endometriosis, the animals were grouped as follows:

Group A was administered injectable water via an intraperitoneal (i.p.) route for 3 days, and then dimethylsulfoxide (DMSO, Merck KGaA, Darmstadt, Germany) via an intragastric (i.g.) route for 20 days, starting on postoperative day 10 of the endometriosis model.

Group B was administered $100 \mathrm{mg} / \mathrm{kg}$ curcumin (Sigma-Aldrich Corporation, St. Louis, USA) per day via an i.g. route. The curcumin was dissolved in DMSO and administered for 20 days, starting on postoperative day 10 of the endometriosis model.

Group $\mathrm{C}$ was administered $100 \mathrm{mg} / \mathrm{kg}$ deferoxamine (Desferal $0.5 \mathrm{~g}$ vials, Novartis AG, Istanbul, Turkey) dissolved in injectable water at 6-hour intervals for 3 days via an i.p. route. Then $100 \mathrm{mg} / \mathrm{kg}$ curcumin per day was administered by an i.g. route for 20 days, starting on postoperative day 10 of the endometriosis model.

A total of 30 animals ( $n=10$ each group) were used to develop the endometriosis model. Three animals in group A, 1 animal in group B and 3 animals in group C died at different stages of the experimental protocol. In addition, 2 animals in group B did not display any sign of ectopic endometriosis at the end of the curcumin treatment. These animals were therefore excluded from the study and 7 animals were used from each group.

After administering the agents, the body weights of the animals were measured, biopsy materials were obtained under ketamine/xylazine anesthesia, and the sizes of the endometriotic-like implants were again measured.

\section{Histological and immunohistochemical techniques}

All eutopic and ectopic materials were fixed in $10 \%$ formaldehyde and embedded in paraffin. The samples were then cut into $5 \mu \mathrm{m}$ sections and stained with hematoxylin and eosin (H \& E) to microscopically confirm whether a model of endometriosis had been created. For immunohistochemical confirmation of the ectopic areas, a monoclonal mouse cytokeratin-7 antibody (5F282:sc-70936, Santa Cruz Biotechnology Inc., Dallas, USA) was used. 
For the immunohistochemical procedures, the slides were deparaffinized in toluene and rehydrated in a graded alcohol series. For antigen retrieval, the slides were boiled for 15 min in a 10-millimolar citrate buffer (pH 6). Subsequently, the sections were immersed in $3 \%$ hydrogen peroxide for $5 \mathrm{~min}$. To block non-specific staining, the slides were incubated in a humidified chamber with $5 \%$ normal horse serum (Vector Laboratories Inc., Burlingame, USA) in Tris-buffered saline (TBS) for $30 \mathrm{~min}$ at room temperature. The excess serum was drained and the sections were incubated with a monoclonal mouse PCNA antibody (F-2, sc-25280, 1 : 300 dilution in TBS; Santa Cruz Biotechnology Inc.) and a monoclonal mouse cytokeratin-7 antibody (Santa Cruz Biotechnology Inc.) at room temperature for $1 \mathrm{~h}$. Biotinylated horse anti-mouse antibody (Vector Laboratories Inc.) was added at a $1: 400$ dilution to both primary antibodies for $30 \mathrm{~min}$. The sections were then incubated with a streptavidin-biotin-peroxidase kit (Vector Laboratories Inc.), and the resultant immunoreactivity was visualized using 3,3-diaminobenzidine tetrahydrochloridedihydrate (DAB; Vector Laboratories Inc.). After the sections were slightly counterstained with hematoxylin, they were mounted with coverslips.

The intensity of proliferating cell nuclear antigen (PCNA) immunoreactivity was evaluated in a semi-quantitative manner using the intensity categories and procedure suggested by Seval et al.: "0, no staining; $1+$, weak but detectable staining; $2+$, moderate or distinct staining; and $3+$, intense staining. For each tissue, a histological score (HSCORE) value was derived by calculating the sum of the percentages of cells that stained in each intensity category and then multiplying that value by the weighted intensity of the staining using the formula HSCORE $=\Sigma P i(i+1)$, where $i$ represents the intensity scores and $P i$ is the corresponding percentage of cells" ${ }^{13}$ Five randomly selected areas on each slide were evaluated under a light microscope at $\times 200$ magnification at different times by 2 investigators who were blind as to the tissue types and sources, and the percentage of cells at each intensity within these areas was determined. The mean value of the 2 investigators' scores was the basis for the statistical calculations.

\section{Atomicabsorption spectrometry (AAS) technique}

Blood taken from the hearts of the animals was kept at $-80^{\circ} \mathrm{C}$, and the blood iron levels were measured using an atomic absorption spectrophotometer.

Iron $(\mathrm{Fe})$ atomic absorption standard solution $(1000 \mathrm{mg} / \mathrm{L})$ was used (Inorganic Ventures, Christiansburg, USA). The Fe concentrations were determined using a Perkin Elmer AAnalyst 800 Atomic Absorption Spectrophotometer (AAS, International Equipment Trading Ltd., Mundelein, USA) with a deuterium lamp and an air/acetylene burner.

The instrumental parameters of the iron element were as follows:
- the hollow cathode lamp was operated at $5 \mathrm{~mA}$ for Fe;

- working wavelength: $248.3 \mathrm{~nm}$;

- slit width: $0.2 \mathrm{~nm}$

One milliliter serum samples obtained from the blood samples stored at $-80^{\circ} \mathrm{C}$ were immersed in water at room temperature until they began to appear homogeneous; a wet solubilization method was then applied. The mineral acids and oxidizing acids used in this method are effective substances for solubilizing both organic and inorganic substances. Solubilization occurs because the acids remove the matrix from the samples. For this purpose, $2 \mathrm{~mL}$ of an $\mathrm{HClO}_{4}-\mathrm{HNO}_{3}$ acid mixture $(1: 6)$ was added to the samples, and these samples were incubated in a sealed polyethylene container overnight. This process produced an exact solubilization of the iron content with the oxidant. The solubilized serum samples were filtered into polyethylene bottles using filter paper, and the filtrates were diluted to a volume of $10 \mathrm{~mL}$ using distilled water. A blank solution was obtained using the same procedure. The iron in the solubilized serum samples was determined using the atomic absorption spectrophotometer. A standard addition technique was used for the AAS determination due to potential interference present in the tested sample.

\section{Statistical analysis}

The statistical analysis was carries out using SPSS 11 software (SPSS Inc., Chicago, USA). The results were expressed as mean \pm standard deviation (SD). In all cases, $\mathrm{p}<0.05$ was considered statistically significant. To evaluate statistically significant differences between the 3 groups, the initial and final body weights were assessed using a paired t-test. An ANOVA test was used to compare the endometriotic-like implants sizes, serum iron levels and PCNA immunoreactivity data in each group.

\section{Results}

\section{Animal weights, measurement of iron in the blood and endometriotic-like implant size}

The body weights of the animals were measured by the same person using the same scale. No significant differences were found between the initial and final weights in any of the 3 groups (Table 1 ).

The blood iron level measurements were repeated 3 times during the experiment. The blood iron levels in the 3 groups were not significantly different $(\mathrm{p}=0.991$; Table 2).

When the measurements of the endometriotic-like implants at the beginning of the experiment were compared, no significant differences were observed between the 3 groups ( $p=0.762$; Table 1$)$. At the end of the experiment, however, the endometriotic-like implant sizes were 
Table 1. Comparison of animal body weights and endometriotic-like implant sizes before and at the end of the experiment

\begin{tabular}{|l|cc|}
\hline Parameters & Group a $(\mathrm{n}=7)$ & Group b $(\mathrm{n}=7)$ \\
\hline Initial body weight $(\mathrm{g})$ & $211.85 \pm 12.32$ & $214.57 \pm 6.67$ \\
Final body weight $(\mathrm{g})$ & $211.28 \pm 12.10$ & $219.28 \pm 6.1$ \\
Endometriotic-like implant size before experiment $\left(\mathrm{mm}^{3}\right)$ & $10.98 \pm 2.69$ & $10.56 \pm 1.75$ \\
Endometriotic-like implant size at the end of the experiment $\left(\mathrm{mm}^{3}\right)$ & $32.89 \pm 5.79$ & $201.28 \pm 23.61$ \\
\hline
\end{tabular}

* compared with group a, $p<0.05$. Values are presented as mean \pm SD.

Table 2. Blood iron levels and proliferating cell nuclear antigen (PCNA) immunoreactivity results

\begin{tabular}{|l|cc|c} 
Parameters & Group a $(\mathrm{n}=7)$ & Group b $(\mathrm{n}=7)$ \\
\hline Blood iron $(\mathrm{mg} / \mathrm{L})$ & $3.23 \pm 0.96$ & $3.22 \pm 0.48$ & $3.18 \pm 0.40$ \\
PCNA immunoreactivity in eutopic endometrium & $111.43 \pm 32.50$ & $100.00 \pm 30.35$ \\
PCNA immunoreactivity in ectopic endometrium & $106.83 \pm 23.41$ & $91.25 \pm 28.63$ \\
\hline
\end{tabular}

* compared with group a, $p<0.05$. Values are presented as mean \pm SD.

significantly reduced in groups b and $\mathrm{c}-$ the curcumin and curcumin + deferoxamine groups - as compared with group a (Table 1).

\section{Histopathological evaluation}

In all 3 groups, the eutopic endometrium was observed to have a normal histological appearance (Fig. 1a). The ectopic endometrium was characterized by the coexistence of both glands and stromal structures and a remarkable surrounding fibrotic connective tissue. Areas within the ectopic endometrium were found to be particularly well vascularized. Glandular epithelia were observed to have an appearance similar to that of the eutopic epithelial glands (Fig. 1b).

Cytokeratin-7 antibodies in the eutopic and ectopic endometrium exhibited immunoreactivity in the glandular epithelial cells (Figs. 1c, 1d).

\section{Immunohistochemical evaluations}

PCNA immunoreactivity was used to assess the proliferation rate of epithelial cells in in the eutopic and ectopic endometria of the control animals, the curcumin-treated
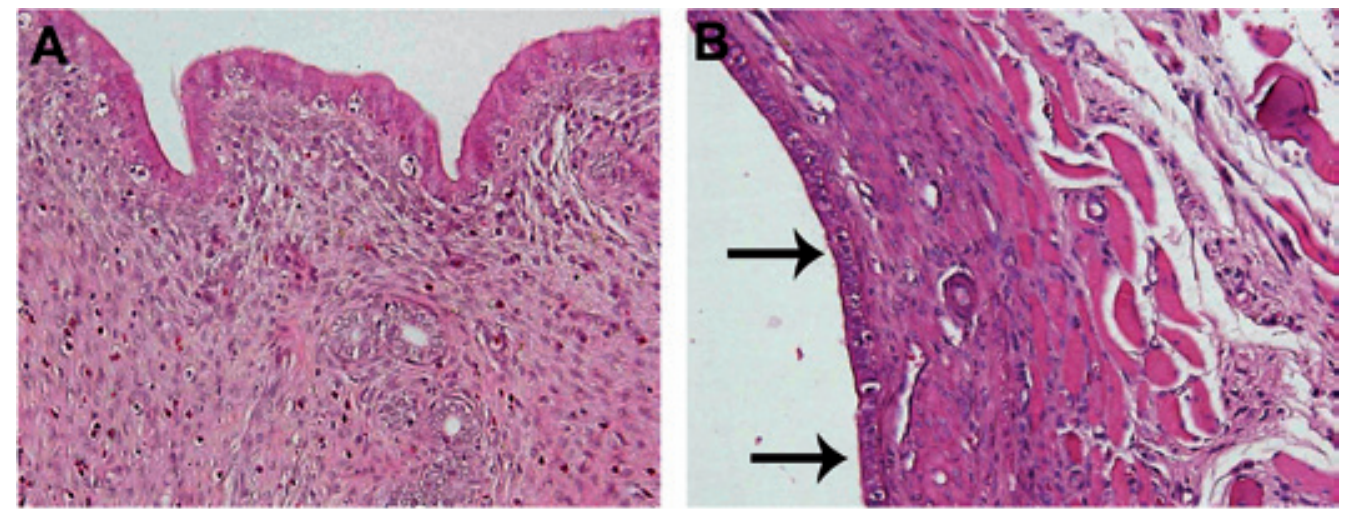

Fig. 1. H\& E and cytokeratin-7 evaluation. Eutopic and ectopic (arrows) endometria stained with $\mathrm{H}$ \& $E$ are shown in $a$ and $b$, respectively. Cytokeratin-7 immunoreactivity in endometrial glandular cells in eutopic and ectopic (arrows) endometria is shown in c and d, respectively. Magnification x200
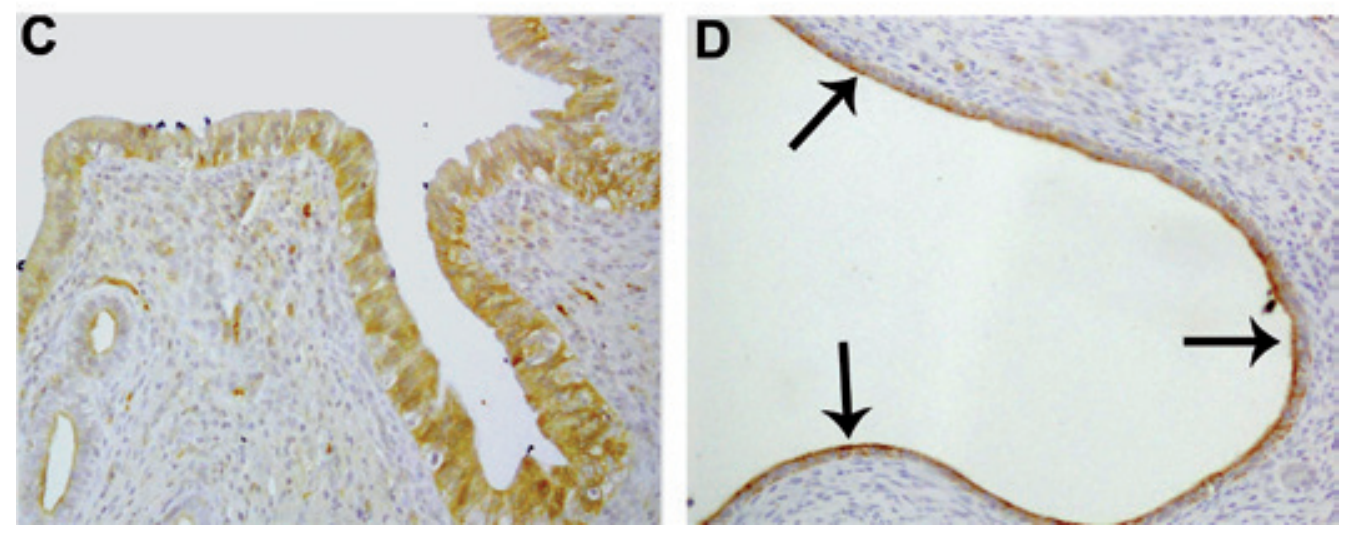

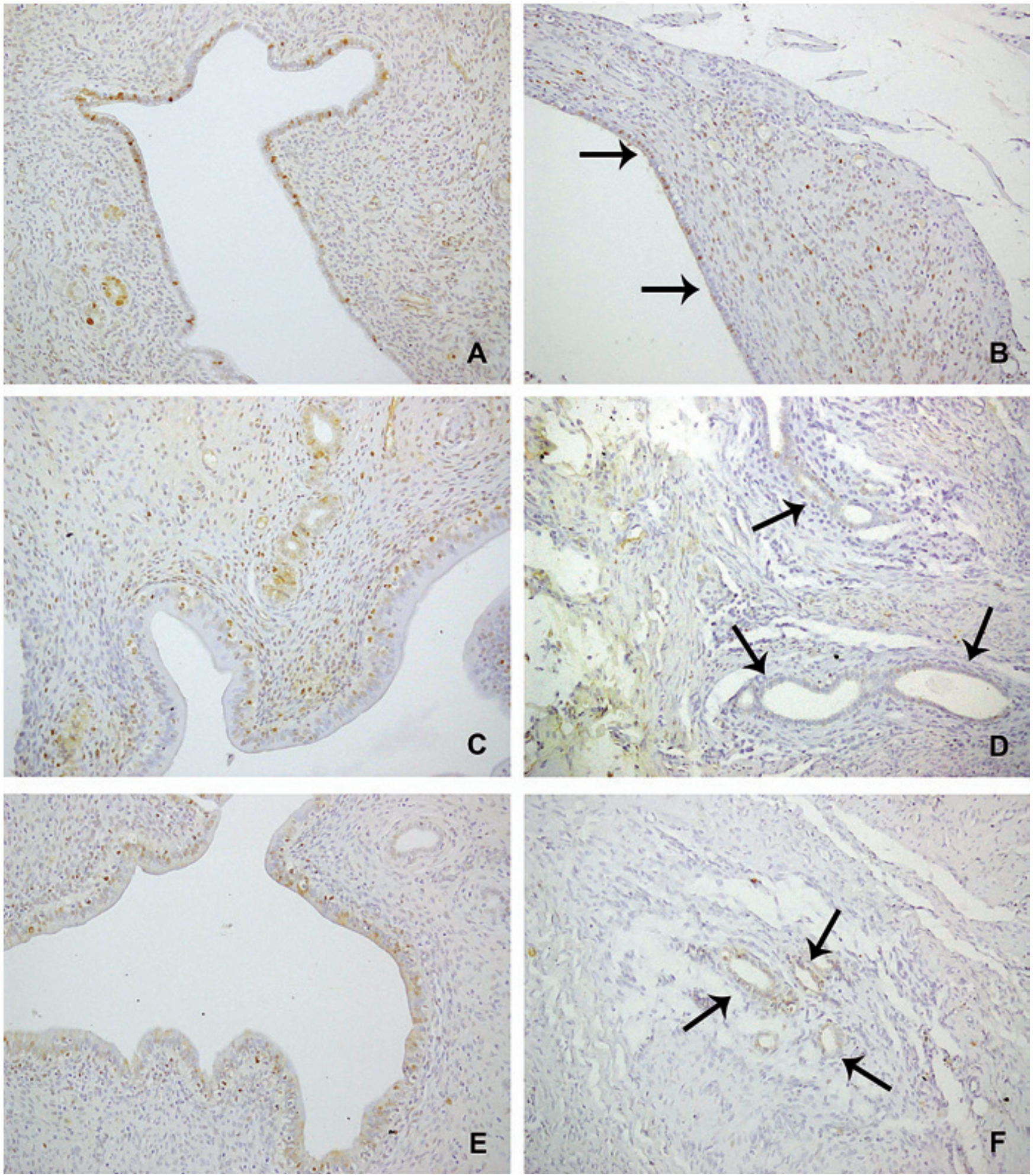

Fig. 2. Proliferating cell nuclear antigen (PCNA) immunoreactivity in eutopic (left column; $a, c$ and e) and ectopic (right column; b, $d$ and f) endometria. No statistically significant differences were found among the groups $\mathrm{a}$, b or $\mathrm{c}$ in eutopic endometria based on PCNA immunoreactivity. However, in ectopic endometria (arrows), PCNA immunoreactivity was significantly higher in group a (b) than in group b (d) and c (f). Magnification x200

animals and the curcumin-with-deferoxamine-treated animals. The values are shown in Table 2.

When the PCNA immunoreactivities in the eutopic endometria of groups $\mathrm{a}, \mathrm{b}$ and $\mathrm{c}$ were compared, no significant differences were found among the groups $(\mathrm{p}=0.454$; Figs. 2a, 2c, 2e and Table 2).
In the ectopic endometrium, PCNA immunoreactivity was significantly lower in groups b and c compared with group a $(\mathrm{p}=0.044$ and $\mathrm{p}=0.033$, respectively; Figs. $2 \mathrm{~b}$, $2 \mathrm{~d}$ and 2f; Table 2). A comparison between the ectopic endometria in groups $b$ and $c$ revealed no significant differences $(\mathrm{p}=1.00$; Figs. $2 \mathrm{~d}-\mathrm{f})$. 


\section{Discussion}

Endometriosis is an increasingly important disease that affects women of reproductive age and can cause infertility. Interest in this disease continues to grow among investigators, as a cure has yet to be found. ${ }^{14,15}$

In the present study, no differences were observed in the 3 groups' pre- and post-experimental body weights (Table 1), which coincides with other researchers' findings. ${ }^{16}$ These results suggest that neither curcumin alone nor curcumin in combination with deferoxamine significantly affects metabolic activity in rats.

Iron levels, which are known to be increased in endometriosis, are considered a possible marker for endometriosis or experimental endometriosis..$^{5-7,17,18}$ The present study measured blood iron levels to determine whether they would change with curcumin alone or in combination with deferoxamine in eutopic and/or ectopic endometrial tissues. This study also investigated whether cell proliferation and the sizes of the endometrial implants or the development of experimental endometriosis would be affected in the groups that received curcumin alone or curcumin + deferoxamine.

In the present study, atomic absorption spectrophotometry showed that the blood iron levels were not significantly different between the 2 treatment groups. These results suggest, at least in part, that neither curcumin nor its combination with deferoxamine affect serum iron levels in a rat endometriosis model. However, Langendonckt et al. suggested that serum iron concentrations were higher than in peritoneal fluid using a colorimetric method in women with endometriosis. ${ }^{18}$

In a study by Zhang et al., a similar endometriosis model was created by inserting large endometrial implants. Subsequently, 3 different doses of curcumin (50, 100 and $150 \mathrm{mg} / \mathrm{kg}$ ) were administered to the animals. Those authors reported that the endometriotic-like implant sizes were significantly decreased in each group. ${ }^{16}$ The results of the group administered $100 \mathrm{mg} / \mathrm{kg}$ of curcumin were the same as those observed in the curcumin group in the present study. Similarly, Hendarto et al. emphasized that curcumin had reduced the size of endometriotic-like implants in experimental endometriosis in mice. ${ }^{19}$ In all 3 studies, including the current findings, curcumin's reduction of the size of endometriotic-like implants is very promising, and warrants further studies aiming toward the development of a novel therapeutic strategy for treatment of endometriosis.

Weiser et al. suggested that curcumin decreases cell proliferation, suppresses the secretion of vascular endothelial growth factor (VEGF) and causes apoptosis in eutopic endometrial cells in women with and without endometriosis. ${ }^{20}$ Recent studies have revealed that curcumin nearly reversed the activity of matrix metalloproteinases (MMP)-2, MMP-3 and MMP-9, and that increasing MMP-2, MMP-3 and MMP-9 levels in patients with en- dometriosis exacerbated the disease. ${ }^{21-23}$ In addition, curcumin, which is also an inhibitor of NF-kB can block all the potential effects of NF- $\mathrm{kB}$ by activating various pathways and increasing the secretion of different proteins in endometriosis. ${ }^{24-27}$

Defrère et al. found that the proliferative activity of epithelial cells in the ectopic endometrium decreased when deferoxamine was applied to nude mice with experimental endometriosis. ${ }^{6}$ They concluded that an iron overload does not affect the occurrence of new lesions. Moreover, it may cause endometriosis to spread by increasing epithelial cell proliferation in these lesions. ${ }^{6}$ According to this view, which is parallel to the present authors' ideas, iron-chelating treatments may be useful for endometriosis, reducing the proliferation of lesions by preventing iron loading in the pelvic cavity. ${ }^{17}$ Although the present authors did not observe further improvement in experimental endometriosis using the curcumin + deferoxamine combination as compared with curcumin alone, future studies examining increases in the dose and timing of this combination may reveal possible additive effects to a significant level when compared with curcumin alone.

\section{Conclusions}

In conclusion, to the best of the authors' knowledge, this is the first experimental endometriosis study demonstrating the combined effect of curcumin and deferoxamine. The findings suggest that the combined use of different iron-chelating agents may lead to a reduction in endometrial implant sizes and cell proliferation when used to treat endometriosis. However, further studies from many different perspectives are required.

\section{References}

1. Kizilay G, Cakmak H, Arici A. Endometriosis and implantation. Balkan Med J. 2007;24:90-97.

2. Ihara T, Uchiide I, Sugamata M. Light and electron microscopic evaluation of antileukotriene therapy for experimental rat endometriosis. Fertil Steril. 2004;81:819-823.

3. Sharpe-Timms KL. Using rats as a research model for the study of endometriosis. Ann N Y Acad Sci. 2002;955:318-327.

4. Rezende $C A L$, Silva $A L$, Junior JLS, Gobbi H, Martins MMF. Experimental model for endometriosis-comparative histological study between the ectopic and eutopic endometrium. Acta Cír Bras. 1997; 12:226-230.

5. Lousse JC, Defrere S, Van Langendonckt A, et al. Iron storage is significantly increased in peritoneal macrophages of endometriosis patients and correlates with iron overload in peritoneal fluid. Fertil Steril. 2009;9:1668-1675.

6. Defrere S, Van Langendonckt A, Vaesen S, et al. Iron overload enhances epithelial cell proliferation in endometriotic lesions induced in a murine model. Hum Reprod. 2006;21:2810-2816.

7. Van Langendonckt A, Casanas-Roux F, Eggermont J, Donnez J. Characterization of iron deposition in endometriotic lesions induced in the nude mouse model. Hum Reprod. 2004;19:1265-1271.

8. Langlois A, Bietiger W, Mandes K, et al. Overexpression of vascular endothelial growth factor in vitro using deferoxamine: A new drug to increase islet vascularization during transplantation. Transplant Proc. 2008;40:473-476. 
9. Wu MH, Chen KF, Lin SC, Lgu CW, Tsai SJ. Aberrant expression of leptin in human endometriotic stromal cells is induced by elevated levels of hypoxia inducible factor-1a. Am J Pathol. 2007;70:590-598.

10. Srichairatanakool S, Thephinlap C, Phisalaphong C, Porter JB, Fucharoen $\mathrm{S}$. Curcumin contributes to in vitro removal of non-transferrin bound iron by deferiprone and desferrioxamine in thalassemic plasma. Med Chem. 2007;3:469-474.

11. Hatcher H, Planalp R, Cho J, Torti FM, Torti SV. Curcumin: From ancient medicine to current clinical trials. Cell Mol Life Sci. 2008;65: 1631-1635.

12. Kim KH, Lee EN, Park JK, et al. Curcumin attenuates TNF-a-induced expression of intercellular adhesion molecule-1, vascular cell adhesion molecule- 1 and proinflammatory cytokines in human endometriotic stromal cells. Phytother Res. 2012;26:1037-1047.

13. Seval Y, Cakmak H, Kayisli UA, Arici A. Estrogen-mediated regulation of p38 mitogen-activated protein kinase (MAPK) in human endometrium. J Clin Endocrinol Metab. 2006;91:2349-2357.

14. Ricci AG, Olivares $C N$, Bilotas $M A$, et al. Natural therapies assessment for the treatment of endometriosis. Hum Reprod. 2013;28:178-188.

15. Nenicu A, Körbel C, Gu Y, Menger MD, Laschke MW. Combined blockade of angiotensin II type 1 receptor and activation of peroxisome proliferator-activated receptor- $\gamma$ by telmisartan effectively inhibits vascularization and growth of murine endometriosis-like lesions. Hum Reprod. 2014;29:1011-1024.

16. Zhang $\mathrm{Y}, \mathrm{Cao} \mathrm{H}, \mathrm{Hu} \mathrm{YY}$, Wang H, Zhang CJ. Inhibitory effect of curcumin on angiogenesis in ectopic endometrium of rats with experimental endometriosis. Int Jour of Mol Med. 2011;27:87-94.

17. Defrère S, Lousse JC, González-Ramos R, Colette S, Donnez J, Van Langendonckt A. Potential involvement of iron in the pathogenesis of peritoneal endometriosis. Mol Hum Reprod. 2008;14:377-85.
18. Van Langendonckt A, Casanas-Roux F, Donnez J. Iron overload in the peritoneal cavity of women with pelvic endometriosis. Fertil Steril. 2002;78:712-718.

19. Hendarto HHH, Kuswojo HKK, Sa'adi AAS, Ramelan WWR, Sudiana IKIKS. The role of curcumin supplementation on implant growth and fertilization result of experimental endometriosis in mice. Fertil Steril. 2010;94:205.

20. Wieser F, Yu J, Park J, Sidell N, Taylor RN. Curcumin suppresses angiogenesis, cell proliferation and induces apoptosis in an in vitro model of endometriosis. Fertil Steril. 2007:88:204-205.

21. Jana S, Rudra DS, Paul S, Snehasikta S. Curcumin delays endometriosis development by inhibiting MMP-2 activity. Indian J Biochem Biophys. 2012;49:342-348.

22. Jana S, Paul S, Swarnakar S. Curcumin as anti-endometriotic agent: Implication of MMP-3 and intrinsic apoptotic pathway. Biochem Pharmacol. 2012;83:797-804.

23. Swarnakar S, Paul S. Curcumin arrests endometriosis by downregulation of matrix metalloproteinase-9 activity. Indian J Biochem Biophys. 2009;46:59-65.

24. Poylin V, Fareed MU, O'Neal P, et al. The NF-Kappaß inhibitor curcumin blocks sepsis-induced muscle proteolysis. Mediators Inflamm. 2008;2008:ID:317851.

25. Kim YS, Ahn Y, Hong MH, et al. Curcumin attenuates inflammatory responses of TNFa-stimulated human endothelial cells. J Cardiovasc Pharmacol. 2007:50:41-49.

26. Veillat V, Lavoie CH, Metz CN, Roger T, Labelle Y, Akoum A. Involvement of nuclear factor- $\mathrm{KB}$ in macrophage migration inhibitory factor gene transcription up-regulation induced by interleukin-1 $\beta$ in ectopic endometrial cells. Fertil Steril. 2009;91:2148-2156.

27. Reinaldo GR, Langendonckt AV, Defrere $S$, et al. Involvement of the nuclear factor- $\mathrm{KB}$ pathway in the pathogenesis of endometriosis. Fertil Steril. 2010;94:1985-1994. 